\title{
Linear Numerical-Magnitude Representations Aid Children's Memory for Numbers
}

Psychological Science 2I(9) |274-128I (c) The Author(s) 2010 Reprints and permission: sagepub.com/journalsPermissions.nav DOI: 10.1 1 177/0956797610378309 http://pss.sagepub.com ๑SAGE

\author{
Clarissa A.Thompson' and Robert S. Siegler ${ }^{2}$ \\ 'The University of Oklahoma and ${ }^{2}$ Carnegie Mellon University
}

\begin{abstract}
We investigated the relation between children's numerical-magnitude representations and their memory for numbers. Results of three experiments indicated that the more linear children's magnitude representations were, the more closely their memory of the numbers approximated the numbers presented. This relation was present for preschoolers and second graders, for children from low-income and middle-income backgrounds, for the ranges 0 through 20 and 0 through I,000, and for four different tasks (categorization and number-line, measurement, and numerosity estimation) measuring numerical-magnitude representations. Other types of numerical knowledge-numeral identification and counting - were unrelated to recall of the same numerical information. The results also indicated that children's representations vary from trial to trial with the numbers they need to represent and remember and that general strategy-choice mechanisms may operate in selection of numerical representations, as in other domains.
\end{abstract}

\section{Keywords}

numbers, numerical representations, memory, estimation, cognitive development

Received 10/27/09; Revision accepted 12/29/09

The modern world is a world of numbers, many of which people need to remember: social security numbers, phone numbers, flight numbers, credit-card numbers, locker-combination numbers, and so on. Memory for numbers is strongly related to school grades and achievement-test scores, which is why it is included on many IQ tests.

The experiments we report here demonstrate a surprising influence on memory for numbers: representations of the numbers' magnitudes. A wide range of behavioral and neuroscience evidence indicates that both children and adults represent numbers on something akin to a mental number line (Ansari, 2008; Case, Okamoto, Henderson, McKeough, \& Bleiker, 1996; Dehaene, 1997). Individual differences in the linearity of numerical-magnitude estimates - the extent to which estimates increase linearly with the number being estimated-are strongly, positively related to children's and preadolescents' arithmetic proficiency, mathematics achievement-test scores, and mathematics course grades (Booth \& Siegler, 2006; Laski \& Siegler, 2007; Schneider, Grabner, \& Paetsch, 2009). The present findings indicate one source of this relation: Accurate numerical representations aid memory for numbers.

Children's numerical representations appear to progress from an initial period in which children do not associate number names with their magnitudes (Le Corre, Van de Walle, Brannon, \& Carey, 2006; Sarnecka \& Carey, 2008), to a period in which number names are associated with their magnitudes via a logarithmically increasing function (Laski \& Siegler, 2007; Siegler \& Booth, 2004), to a period in which number names are associated with their magnitudes via a linearly increasing function (Opfer \& Thompson, 2008; Thompson \& Opfer, 2008). The main improvement occurs during preschool for the numbers 0 through 10 (Berteletti, Lucangeli, Piazza, Dehaene, \& Zorzi, 2010; Le Corre \& Carey, 2007; Opfer, Thompson, \& Furlong, in press), between kindergarten and second grade for 0 through 100 (Geary, Hoard, Nugent, \& Byrd Craven, 2008; Siegler \& Booth, 2004), between second and fourth grade for 0 through 1,000 (Booth \& Siegler, 2006), and between third and sixth grade for 0 through 10,000 (Thompson \& Opfer, in press).

Similar patterns of developmental and individual differences have been found on a variety of tasks measuring

Corresponding Author:

Clarissa A.Thompson, Department of Psychology, 455 W. Lindsey St., Dale

Hall Tower \# 727, Norman, OK 73019

E-mail: cat3@ou.edu 
numerical-magnitude representations. For example, Booth and Siegler (2006) found that for the 0-through-1,000 range, three types of estimation-number line, measurement, and numerosity - undergo parallel transitions from predominant use of a logarithmic representation to predominant use of a linear representation between second and fourth grade and that linearity of individual children's estimates is highly correlated across the three tasks. These relations are not limited to this age or numerical range or to estimation tasks; similar developmental and individual differences have been found for numerical categorization between kindergarten and second grade for the 0-through-100 range (Laski \& Siegler, 2007).

In the present study, we tested four theoretical predictions regarding the relation between numerical representations and memory for numerical content. First, use of a linear representation of numerical magnitudes should be associated with superior memory for numbers. As illustrated in Figure 1, for the largest $85 \%$ of numbers, the subjective distance between successive numbers is greater in a linear than in a logarithmic representation. This greater mental distance between successive numbers should increase the discriminability of numbers and thus improve recall accuracy.

Second, other numerical processes, such as counting and numeral identification, should be less closely related to memory for numbers. If magnitudes are indeed the gist of numbers
(Siegler \& Ramani, 2009), then tasks that directly measure understanding of numerical magnitudes should be more closely related to memory for numbers than other numerical tasks are.

Third, memory for small numbers should be superior to memory for larger ones. Within a logarithmic representation, small numbers are spaced further apart than large ones (see Fig. 1). Older children and adults who are able to use linear representations of numerical magnitude also use logarithmic representations when that is appropriate or convenient (Banks \& Coleman, 1981; Holyoak \& Mah, 1982). Given the generally adaptive quality of strategy choices (Siegler, 1996), it seemed likely that even children who can use linear representations will use logarithmic representations to remember small numbers. This prediction (and the next one as well) rests on the assumption that children who use linear representations on numerical-magnitude estimation tasks also possess logarithmic representations and use them if there is reason to do so, but that children who use logarithmic representations on tasks for which linear representations would yield greater accuracy cannot use linear representations, at least for larger numbers.

Fourth (and least intuitive), large numbers should be recalled more accurately by children who use linear representations on tasks assessing representations of numerical magnitudes, but small numbers should be recalled equally accurately

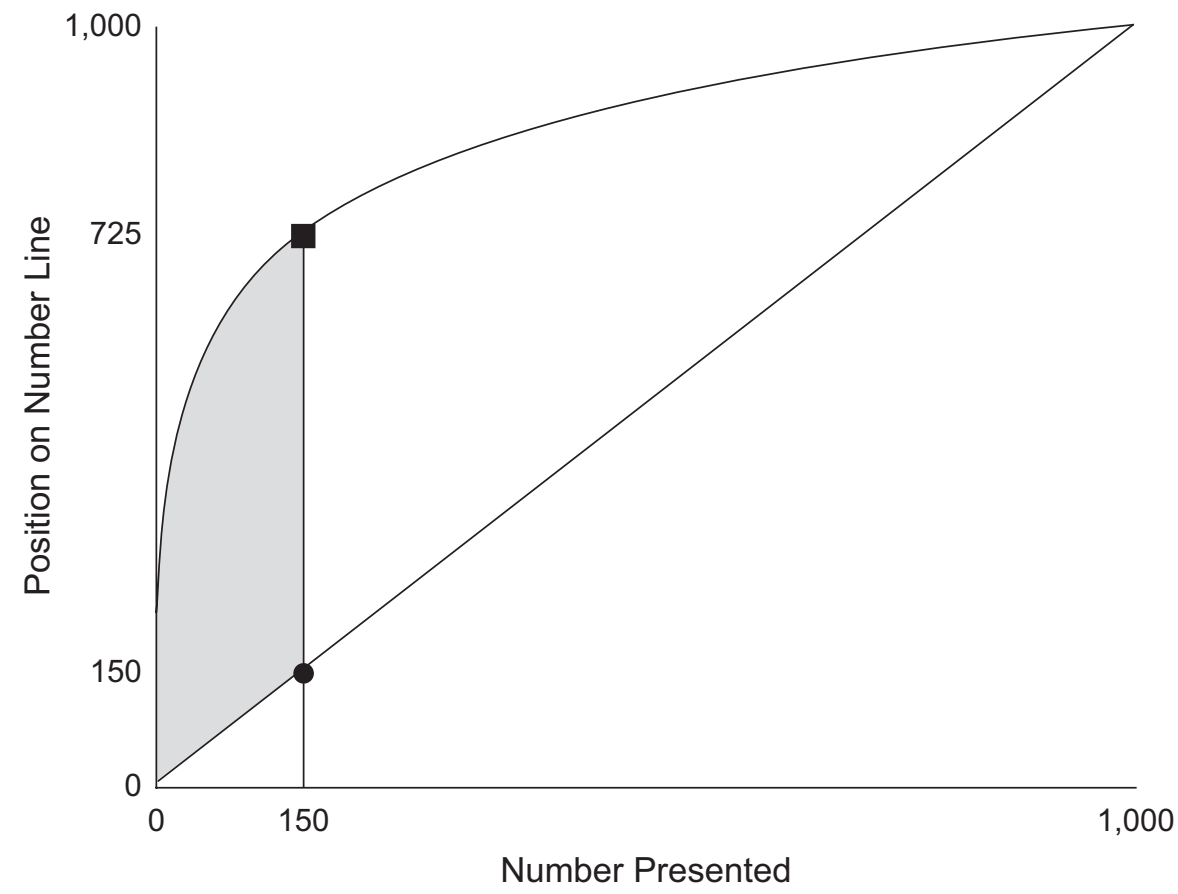

Fig. I. Logarithmic and linear representations of numerical magnitude. When logarithmic and linear functions are constrained to pass through 0 and I,000, the distance between the values generated by the two functions (i.e., the difference between the predicted number-line estimates) is greatest at 150. This means that the values generated by the logarithmic function increase more than those generated by the linear function between each successive pair of numbers up to 150, but the values generated by the logarithmic function increase less than those generated by the linear function between each successive pair of numbers above I50. Thus, numbers below 150 are more discriminable in the logarithmic representation, and numbers above I50 are more discriminable in the linear representation. 
regardless of whether children use logarithmic or linear representations on tasks assessing magnitude representations. In a distribution from 0 through 1,000, in which the linear and logarithmic functions must both pass through 0 and 1,000, numbers above 150 are spaced further apart in the linear function than in the logarithmic one (Fig. 1). Therefore, children who use linear representations should show superior recall of numbers above 150 (relative to children who use logarithmic representations), because those numbers' magnitudes are more distinct within the linear representation. In contrast, it is useful to rely on a logarithmic representation for purposes of remembering numbers between 0 and 150 . The reason is that numbers below 150 are spaced further apart in the logarithmic function, which yields more discriminable, and presumably easier-to-remember, magnitude representations. Therefore, we assumed that both groups of children would use logarithmic representations for remembering numbers below 150. This led to the prediction that the two groups would recall numbers below 150 equally well. Note that although greater exposure to smaller numbers might explain those numbers being more memorable, it does not predict this number-size-byrepresentation interaction.

\section{Experiment I}

Experiment 1 tested our first and third predictions: that individual differences in the linearity of numerical-magnitude representations are related to individual differences in children's memory for numerical content, and that smaller numbers are remembered better than larger ones.

\section{Method}

Participants. Participants were 18 children (mean age $=5.17$ years, $S D=0.40 ; 56 \%$ males, $44 \%$ females; $94 \%$ Caucasians) attending two prekindergartens near Pittsburgh, Pennsylvania. The school districts considered these children at risk for school failure, in most cases because of low incomes. The experimenter was a Caucasian female.

Tasks. Numerical-magnitude representations were assessed with number-line estimation and numerical categorization tasks. Memory for numbers was assessed with a numerical recall task.

For the number-line estimation task, the children received sheets of paper, each of which had a 20 -cm horizontal line with " 0 " below the left end and " 20 " below the right end. After being shown where 0 and 20 went, the children were asked to estimate the positions of 1 through 19, presented in random order, one number per number line.

For the numerical categorization task, the children were presented three equal-sized boxes, labeled "small," "medium," and "big," respectively. The experimenter said that 0 was a small number and should go in the box labeled "small" and that 20 was a big number and should go in the box labeled "big." Then, the children were orally presented the numbers 1 through 19 in random order; they were asked whether each number was small, medium, or big and asked to point to the box representing where each number belonged. This task has been found to be a useful measure of young children's numerical-magnitude representations (Laski \& Siegler, 2007).

For our assessment of numerical recall, the children listened to six short vignettes (adapted from Brainerd \& Gordon, 1994). Each included two "small" numbers (1, 2, 3, or 4), two "medium" numbers $(9,10,11$, or 12$)$, or two "big" numbers $(17,18,19$, or 20). After each vignette, the children named four cartoon characters and then were asked to recall the numbers in the story. For example, one story stated, "Colleen washes the dishes at a restaurant. She washed $N_{1}$ forks and $N_{2}$ plates." After the interpolated task, the children were asked, "How many forks did Colleen wash? How many plates did Colleen wash?" Table S1 in the Supplemental Material available online presents all the vignettes and questions. Numbers were presented in counterbalanced order within vignettes, and each number was presented equally often with each vignette.

\section{Results}

Recall was measured by number correct, by percentage of variance accounted for by the best-fitting linear function relating the number that was presented to the number that was recalled $\left(R_{\text {lin }}^{2}\right)$, and by percentage of absolute error (PAE) on each trial. PAE was calculated as follows: (|recalled number presented number|) $/ 20 \times 100(20$ was used as the denominator because the numbers being estimated ranged from 0 through 20). Quality of numerical-magnitude representations was measured by PAE and $R_{\text {lin }}^{2}$ on the number-line estimation task and by $R_{\text {lin }}^{2}$ on the numerical categorization task.

As predicted, numerical recall and numerical-magnitude representations were related (Table 1). This was especially the case for the linearity-of-recall measure, which was correlated $(r=|.56|$ or $r=|.57|)$ with all three measures of numericalmagnitude knowledge. (Table 1 also presents the strong correlations among the tasks measuring linearity of numericalmagnitude representations.)

Also as predicted, the smaller the number, the more likely it was to be recalled correctly, $r=-.60, p<.05$. Children correctly remembered far more small numbers $(39 \%)$ than medium ones $(6 \%), t(17)=3.69, p<.01, d=0.98$, or large ones $(17 \%), t(17)=2.20, p<.05, d=0.54$. Correct recall of medium and large numbers did not differ significantly.

Analyses of PAE indicated that distance of the recalled number from the correct answer also varied with numerical size, $F(2,34)=3.43, p<.05, \eta^{2}=.17$. PAE tended to be smaller for small than for large numbers ( $22 \%$ vs. $40 \%), t(17)=$ $2.00, p=.06, d=0.46$; PAE for medium-size numbers was in between $(28 \%)$. 
Table I. Correlations Among Tasks and Measures in Experiment I

\begin{tabular}{lcccc}
\hline Task and measure & Memory: PAE & Memory: $R_{\text {lin }}^{2}$ & Number line: PAE & Number line: $R_{\text {lin }}^{2}$ \\
\hline Memory: $R_{\text {lin }}^{2}$ & $-.78^{* *}$ & & & \\
Number line: PAE & .25 & $-.57^{*}$ & & \\
Number line: $R_{\text {lin }}^{2}$ & -.39 & $.56^{*}$ & $-.80^{* *}$ & \\
Categorization: $R_{\text {lin }}^{2}$ & $-.57^{*}$ & $.57^{*}$ & $-.54 *$ & $.56 *$ \\
\hline
\end{tabular}

Note: $R_{\text {lin }}^{2}=$ percentage of variance accounted for by the best-fitting linear function relating the number that was presented to the number that was estimated or recalled or the category that was chosen; $\mathrm{PAE}=$ percentage of absolute error. $*_{p}<.05 .{ }^{* *} p<.01$.

\section{Experiment 2}

Results of Experiment 1 were consistent with the hypothesis that the linearity of numerical-magnitude representations is closely related to memory for numerical information. However, it was possible that any type of numerical knowledge would be equally strongly related to numerical memory. To test this alternative hypothesis, in Experiment 2, we presented not only the memory and magnitude representation tasks from Experiment 1 , but also two other numerical tasks that did not directly measure magnitude representations: numeral identification and counting. Our prediction was that counting and numeral identification would be less strongly correlated with numerical recall than would the measures of magnitude representation. Experiment 2 also tested whether the Experiment 1 findings held for a different population - children from middle-income rather than low-income backgrounds - and for a stimulus set in which the numbers that needed to be remembered were continuously distributed throughout the 0-through-20 range, rather than falling into discrete categories of small, medium, and large.

\section{Method}

Nineteen preschoolers (mean age $=4.96$ years, $S D=0.52 ; 68 \%$ male, 32\% female; 68\% Caucasian, 32\% Asian American) recruited from three preschools serving middle-income populations in the Pittsburgh, Pennsylvania, area participated. They were presented the same number-line estimation, numerical categorization, and number-recall tasks as in Experiment 1, the only differences being that the numbers were $1,2,3,5,7$, $9,10,11,13,15,17$, and 19 , and all numbers were paired with all other numbers in the vignettes. Also presented were a counting task that involved counting 20 blue poker chips attached to a thin strip of cardboard and a numeral identification task that involved reading Arabic numerals (the same ones used for the estimation, categorization, and recall tasks), presented in random order on flash cards. Analyses were conducted on the percentage of numbers between 1 and 20 counted before the first error and the percentage of correctly identified numerals. The experimenter was a Caucasian female.

\section{Results}

Greater linearity of responses on the tasks that measured numerical-magnitude representations (number-line estimation and categorization) was associated with superior numerical recall, as measured by both PAE and $R_{\text {lin }}^{2}$ (Table 2 ). In contrast, performance on both tasks that measured other types of numerical knowledge (counting and numeral identification) was not correlated with performance on either measure of

Table 2. Correlations Among Tasks and Measures in Experiment 2

\begin{tabular}{|c|c|c|c|c|c|c|}
\hline Task and measure & Memory: PAE & Memory: $R_{\text {lin }}^{2}$ & Number line: PAE & Number line: $R_{\text {lin }}^{2}$ & Categorization: $R_{\text {lin }}^{2}$ & $\begin{array}{c}\text { Counting: \% } \\
\text { correct }\end{array}$ \\
\hline Memory: $R_{\text {lin }}^{2}$ & $-.92 * *$ & & & & & \\
\hline Number line: $R_{\text {lin }}^{2}$ & $-.47^{*}$ & $.53 *$ & $-.82 * *$ & & & \\
\hline Categorization: $R_{\text {lin }}^{2}$ & $-.68 * *$ & $.66 * *$ & $-.7 \mid * *$ & $.78 * *$ & & \\
\hline
\end{tabular}

Note: $R_{\text {lin }}^{2}$ = percentage of variance accounted for by the best-fitting linear function relating the number that was presented to the number that was estimated or recalled or the category that was chosen; PAE = percentage of absolute error. Only 18 participants contributed data for $R_{\text {lin }}^{2}$ for memory because I participant answered " 5 " on every trial. For all other correlations, 19 participants contributed data.

$* p<.05 . * * p .01$. 
numerical recall. Moreover, the three measures of numericalmagnitude representations were more strongly correlated with each other $(r \mathrm{~s}=|.71|-|.82|)$ than any of them were correlated with either counting or numeral identification $(r \mathrm{~s}=|.11|-|.53|)$ The greater relation between the measures of linearity of numerical-magnitude representations (including linearity of categorization) and recall (relative to the relation between other types of numerical knowledge and recall) was predicted, but the lack of any significant correlation between numeral identification and recall was not expected and differed from findings in another recent study (Berteletti et al., 2010). Table 2 presents the correlations among all tasks and measures used in the experiment.

As in Experiment 1, the larger the number, the less likely it was to be recalled correctly, $r=-.81, p<.01$. Number of answers recalled correctly was greater for the smaller half of numbers than for the larger half $(37 \%$ vs. $23 \%), t(18)=2.45$, $p<.05, d=0.56$, and the numbers recalled were closer to the correct answer when the answer was small $(\mathrm{PAE}=17 \%$ vs. $27 \%), t(18)=2.48, p<.05, d=0.54$.

These results replicated the Experiment 1 findings that linearity of numerical-magnitude representations are significantly related to numerical recall. Experiment 2 also showed that this relation is not limited to low-income children or to stimuli clustered into qualitatively distinct groups of numbers, and demonstrated that other numerical competencies are not as closely related to recall of numerical information.

\section{Experiment 3}

Experiment 3 tested our fourth prediction: that large numbers are recalled more accurately by children who use linear representations of numerical magnitudes than by children who use logarithmic representations, but that small numbers are recalled equally accurately by the two groups. Children in the two groups were expected to remember numbers from 0 through 150 equally well, because both would most often rely on the logarithmic representation for remembering those numbers. In contrast, children who used linear representations of numerical magnitudes were expected to recall numbers greater than 150 more accurately than the other group, because linear representations, on which those children would more often rely, make those larger numbers more discriminable.

A second issue addressed in Experiment 3 concerned whether children's memory for numerical information focuses on the gist of the numbers or is literal. If children extract the gist of numbers, they should more often correctly remember the $100 \mathrm{~s}$ value in three-digit numbers than the $10 \mathrm{~s}$ or $1 \mathrm{~s}$ values.

A third issue involved the generality of the effects observed in Experiments 1 and 2. Would the relation between numericalmagnitude representations and memory for numerical information hold with older children (7- and 8-year-olds), with a wider range of numbers ( 0 through 1,000), and for two additional tasks that have been used previously to measure numerical-magnitude representations (measurement estimation and numerosity estimation; Booth \& Siegler, 2006)? If these two tasks and the number-line task all measure numericalmagnitude representations, then similar findings should emerge for all of them.

\section{Method}

Participants. Participants were 127 second graders (mean age $=8.22$ years, $S D=0.44 ; 59 \%$ males, $41 \%$ females; $91 \%$ Caucasian) who attended elementary schools near Pittsburgh, Pennsylvania. Roughly $30 \%$ of the children were eligible for free or reduced-cost lunches. The experimenters were two Caucasian females.

Tasks. The children first performed, in random order, the number-line, measurement estimation, and numerosity estimation tasks used in Booth and Siegler (2006) to assess representations of numerical magnitudes. The specific numbers presented in all three tasks were $2,5,18,34,56,78,100,122$, $147,150,163,179,246,366,486,606,722,725,738,754$, 818 , and 938. The number-line task was like that used in Experiments 1 and 2, except for having "1,000," rather than " 20 ," at the right end and involving estimation of different numbers. On the measurement estimation task, children saw a short line labeled "1 zip," a long line labeled "1,000 zips," and a number indicating the length of a line (in zips) that they should draw. They drew a line to approximate the desired length. On the numerosity estimation task, children saw a computer screen that depicted one box with 0 dots, one with 1,000 dots, and a third, initially empty, box that could be filled to the desired extent by placing the cursor in the "increase" box or the "decrease" box and holding down the mouse until the desired number of dots was reached.

After completing these three tasks, the children were given the numerical recall task, which was like that used in Experiments 1 and 2, except that each story involved three "small numbers" $(5,18,53,79,164$, or 237$)$, three "medium numbers" $(419,487,524,548,625$, or 632$)$, or three "big numbers" $(725,759,817,846,938$, or 962$)$.

\section{Results}

As predicted, individual differences in recall of numbers were substantially correlated with individual differences on all three tasks measuring numerical-magnitude representations (Table 3). This relation was present regardless of whether performance on each task was measured by linearity or absolute error. Individual differences in performance on the three tasks used to assess numerical-magnitude representations were also closely related to each other (Table 3).

To test whether the interaction of numerical representation and number size influenced recall, we conducted a separate 2 (better-fitting representation: logarithmic or linear) $\times 2$ 
Table 3. Correlations Among Tasks and Measures in Experiment 3

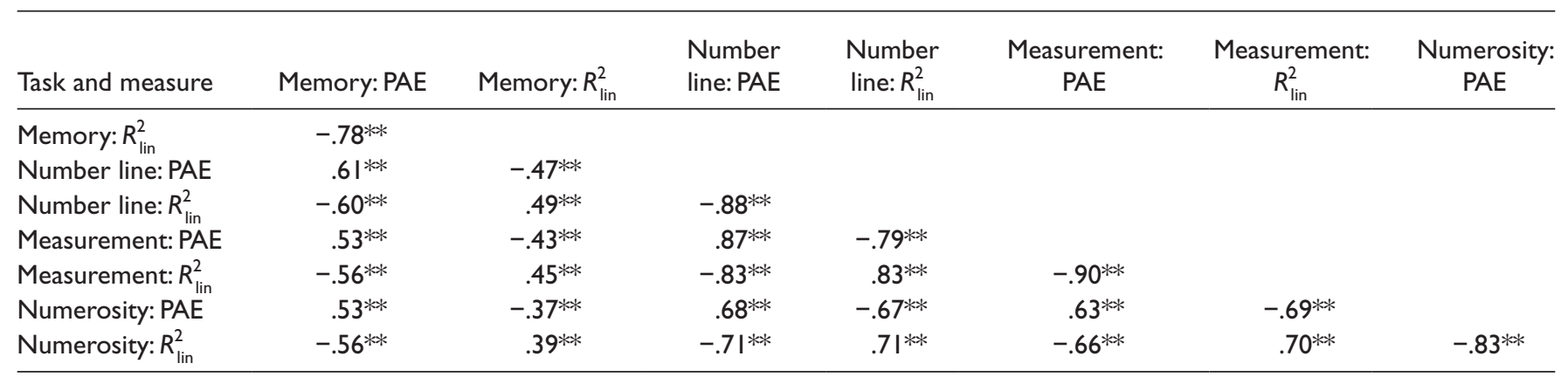

Note: $R_{\text {lin }}^{2}=$ percentage of variance accounted for by the best-fitting linear function relating the number that was presented to the number that was estimated or recalled; $\mathrm{PAE}=$ percentage of absolute error. $* * p<.01$.

(number size: below or above 150) analysis of variance for each of the three tasks assessing numerical representations: the number-line, measurement estimation, and numerosity estimation tasks. As shown in Figure 2, the pattern was highly similar on all three tasks. When the number-line task was used to assess numerical-magnitude representations, recall accuracy (PAE) showed main effects for better-fitting representation, $F(1,125)=23.29, p<.001, \eta^{2}=.16$, and number size, $F(1$, $125)=136.43, p<.001, \eta^{2}=.51$, as well as a representationby-number-size interaction, $F(1,125)=5.75, p<.05, \eta^{2}=.02$. When the measurement estimation task was used to assess numerical representations, recall accuracy again showed main effects for better-fitting representation, $F(1,125)=10.65, p<$ $.01, \eta^{2}=.08$, and number size, $F(1,125)=87.35, p<.001, \eta^{2}=$ .40 , as well as a representation-by-number-size interaction, $F(1$, $125)=7.44, p<.05, \eta^{2}=.03$. Finally, when the numerosity estimation task was used, there were also main effects for better-fitting representation, $F(1,125)=6.31, p<.01, \eta^{2}=.05$, and number size, $F(1,125)=112.75, p<.001, \eta^{2}=.46$, and an interaction of the two, $F(1,125)=4.90, p<.05, \eta^{2}=.02$.

The interactions in all three analyses were of the predicted form. For numbers above 150, children who used linear representations showed superior recall relative to children who used logarithmic representations - number-line estimation (PAEs $=21 \%$ vs. $32 \%): F(1,125)=24.93, p<.001, d=0.86$; measurement estimation (PAEs $=19 \%$ vs. $29 \%): F(1,125)=16.71, p<.001$, $d=0.85$; and numerosity estimation (PAEs $=21 \%$ vs. $29 \%$ ): $F(1,125)=10.16, p<.005, d=0.64$. In contrast, for numbers below 150 , no between-group difference in recall was present for any of the three tasks used to assess numerical representationsnumber-line estimation $(\mathrm{PAEs}=7 \%$ vs. $11 \%): F(1,125)=3.72$, $p>.05$; measurement estimation (PAEs $=8 \%$ vs. $9 \%$ ): $F<1$; and numerosity estimation (PAEs $=8 \%$ vs. $9 \%$ ): $F<1$.

Children were considerably more likely to remember the 100 s digits than the 10 s or 1 s digits, $(28 \%, 15 \%$, and $13 \%$ correct recall, respectively), $\chi^{2}(2, N=127)=168.94, p<.001$.
Number Line

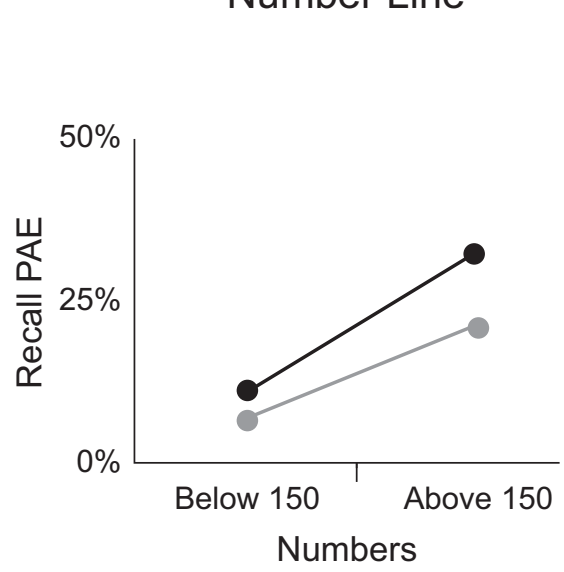

Measurement

- Linear Best Fit $\quad$ Logarithmic Best Fit

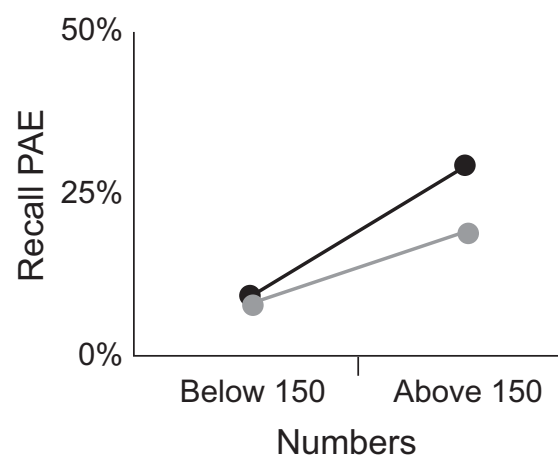

Numerosity

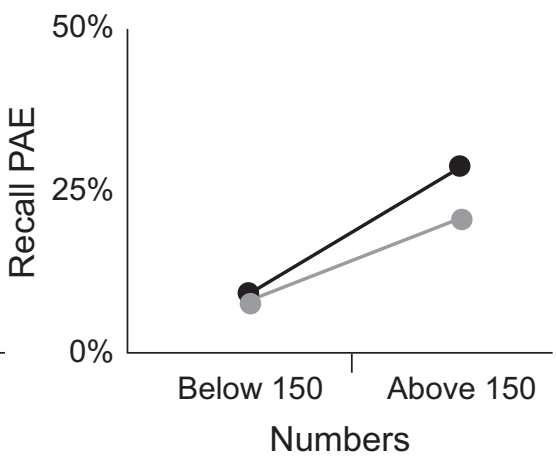

Fig. 2. Recall accuracy (percentage of absolute error, or PAE) as a function of better-fitting numerical-magnitude representation (linear or logarithmic) and number size (below or above 150) for the three tasks used to assess numerical-magnitude representations in Experiment 3: number-line estimation (left), measurement estimation (middle), and numerosity estimation (right). 
This finding supports Brainerd and Gordon's (1994) conclusion that second graders' numerical memory focuses on numbers' gist rather than their verbatim form.

\section{Discussion}

Evidence from these three experiments indicates that children who generally use linear representations of numerical magnitude recall numbers better than those who generally use logarithmic representations. Experiment 1 demonstrated that low-income prekindergartners who produced more linear and more accurate patterns of responses for numbers in the 0 -through-20 range on two measures of numerical-magnitude representations - number-line estimation and numerical categorization - recalled numbers closer in magnitude to the numbers presented within vignettes. Experiment 2 replicated these findings with preschoolers from middle-income backgrounds and demonstrated that numerical tasks that did not assess magnitude understanding showed weaker relations to recall. Experiment 3 extended the findings to older children, a wider numerical range, and two additional measures of numericalmagnitude representations, and also indicated that second graders recall the gist of three-digit numbers (the 100s value) more often than the less significant values (the 10s and 1s values).

Data from Experiment 3 were especially compelling, as they yielded a predicted interactive effect of numerical representation and number size on recall: Differences in recall between children who generally used linear representations and those who generally used logarithmic representations on tasks assessing numerical-magnitude representations were specific to the range of numbers in which the spacing between successive numbers is greater within the linear than within the logarithmic function. This prediction followed straightforwardly from the view that all children would use the logarithmic representation when trying to recall small numbers, but that children who could also use the linear representation would be at an advantage in recalling large numbers, for which the spacing in the linear representation is greater.

Our interpretation of this interaction assumes that children can use logarithmic representations to remember small numbers and linear representations to remember large numbers. This assumption is consistent with Siegler and Opfer's (2003) finding that second graders represent the numbers 0 through 100 linearly in a 0 -through- 100 context and logarithmically in a 0 -through-1,000 context. To further test this assumption, we asked David Geary to reanalyze his trial-by-trial assessments of 289 first graders' number-line representations on a 0-through-100 number-line estimation task (Geary, Hoard, Byrd-Craven, Nugent, \& Numtee, 2007; Geary et al., 2008). Our goal was to test the prediction that children used logarithmic representations more often with smaller numbers. Geary's reanalysis supported the prediction (D.C. Geary, personal communication, September 30, 2009). Children used logarithmic representations of numerical magnitude more often with numbers in the range where the spacing between numbers is greater in the logarithmic than in the linear function (numbers below 15) than they used such representations with larger numbers $(69 \%$ vs. $49 \%), F(1,288)=120.69, p<.001$, $d=0.70$. Greater use of the logarithmic representation for smaller numbers was apparent in our data for all three tasks in Experiment 3, which involved the numbers 0 through 1,000number-line estimation $(52 \%$ vs. $37 \%): t(126)=6.13$, $p<.001, d=0.52$; measurement estimation (63\% vs. $46 \%)$ : $t(126)=9.50, p<.001, d=0.91$; and numerosity estimation $(61 \%$ vs. $47 \%): t(126)=6.12, p<.001, d=0.56$.

The same effect was found in our Experiment 3 data when the analysis was limited to children whose overall estimation pattern on the task was better fit by the linear function. On all three tasks, these children used the logarithmic representation on a higher percentage of trials when the number was below 150 than when it was above 150 - number-line estimation $(38 \%$ vs. $23 \%): t(71)=4.70, p<.001, d=0.57$; measurement estimation ( $38 \%$ vs. $10 \%): t(36)=13.45, p<.001, d=2.25$; and numerosity estimation ( $49 \%$ vs. $34 \%): t(48)=4.48, p<$ $.001, d=0.66$ (see Trial-by-Trial Analysis in the Supplemental Material available online).

Our predictions and findings regarding heightened reliance on logarithmic representations for small numbers among children whose overall representation is linear suggest that the development of numerical representations involves trial-bytrial variability, adaptive choice among representations, and knowledge-driven change like that described in overlappingwaves theory (Siegler, 1996, 2006). Within this theory, representations and strategies that are generally less effective continue to be used in specific situations in which they are effective. The mechanism that produces these adaptive choices is viewed as unconscious, and its workings have been illustrated in computer simulations that generate strategy choices highly similar to children's (Siegler \& Araya, 2005; Siegler \& Shipley, 1995). The present data suggest that similar choice and learning mechanisms contribute to the development of numerical representations.

\section{Acknowledgments}

We thank Callie Hammond for data collection and coding and David Geary for his data reanalysis. We also thank administrators, teachers, parents, and students at Washington Park and McGuffey Pre-K programs; Stepping Stones, Small Wonders, and Crafton Heights preschools; and South Central, Wylandville, Borland Manor, and Bentworth elementary schools.

\section{Declaration of Conflicting Interests}

The authors declared that they had no conflicts of interest with respect to their authorship or the publication of this article.

\section{Funding}

This research was supported by Department of Education Grants R305A080013 and R305H050035. 


\section{Supplemental Material}

Additional supporting information may be found at http://pss.sagepub .com/content/by/supplemental-data

\section{References}

Ansari, D. (2008). Effects of development and enculturation on number representation in the brain. Nature Reviews Neuroscience, 9, 278-291.

Banks, W.P., \& Coleman, M.J. (1981). Two subjective scales of numbers. Perception \& Psychophysics, 29, 95-105.

Berteletti, I., Lucangeli, D., Piazza, M., Dehaene, S., \& Zorzi, M. (2010). Numerical estimation in preschoolers. Developmental Psychology, 46, 545-551.

Booth, J.L., \& Siegler, R.S. (2006). Developmental and individual differences in pure numerical estimation. Developmental Psychology, 41, 189-201.

Brainerd, C.J., \& Gordon, L.L. (1994). Development of verbatim and gist memory for numbers. Developmental Psychology, 30, 163-177.

Case, R., Okamoto, Y., Henderson, B., McKeough, A., \& Bleiker, C. (1996). Exploring the macrostructure of children's central conceptual structures in the domains of number and narrative. Monographs of the Society for Research in Child Development, 61(1-2), 59-82.

Dehaene, S. (1997). The number sense: How the mind creates mathematics. New York: Oxford University Press.

Geary, D.C., Hoard, M.K., Byrd-Craven, J., Nugent, L., \& Numtee, C. (2007). Cognitive mechanisms underlying achievement deficits in children with mathematical learning disability. Child Development, 78, 1343-1359.

Geary, D.C., Hoard, M.K., Nugent, L., \& Byrd Craven, J. (2008). Development of number line representations in children with mathematical learning disability. Developmental Neuropsychology, 33, 277-299.

Holyoak, K.J., \& Mah, W.A. (1982). Cognitive reference points in judgments of symbolic magnitude. Cognitive Psychology, 14, 328-352.

Laski, E.V., \& Siegler, R.S. (2007). Is 27 a big number? Correlational and causal connections among numerical categorization, number line estimation, and numerical magnitude comparison. Child Development, 76, 1723-1743.

Le Corre, M., \& Carey, S. (2007). One, two, three, four, nothing more: An investigation of the conceptual sources of the verbal counting principles. Cognition, 105, 395-438.

Le Corre, M., Van de Walle, G., Brannon, E.M., \& Carey, S. (2006). Re-visiting the competence/performance debate in the acquisition of the counting principles. Cognitive Psychology, 52, 130-169.
Opfer, J.E., \& Thompson, C.A. (2008). The trouble with transfer: Insights from microgenetic changes in the representation of numerical magnitude. Child Development, 79, 790-806.

Opfer, J.E., Thompson, C.A., \& Furlong, E. (in press). Early developments of spatial-numeric associations: Evidence from spatial and quantitative performance of preschoolers. Developmental Science.

Sarnecka, B.W., \& Carey, S. (2008). How counting represents number: What children must learn and when they learn it. Cognition, 108, 662-674.

Schneider, M., Grabner, R.H., \& Paetsch, J. (2009). Mental number line, number line estimation, and mathematical school achievement: Their interrelations in Grades 5 and 6. Journal of Educational Psychology, 101, 359-372.

Siegler, R.S. (1996). Emerging minds: The process of change in children's thinking. New York: Oxford University Press.

Siegler, R.S. (2006). Microgenetic analyses of learning. In W. Damon \& R.M. Lerner (Series Eds.) \& D. Kuhn \& R.S. Siegler (Vol. Eds.), Handbook of child psychology: Vol. 2. Cognition, perception, and language (6th ed., pp. 464-510). Hoboken, NJ: Wiley.

Siegler, R.S., \& Araya, R. (2005). A computational model of conscious and unconscious strategy discovery. In R.V. Kail (Ed.), Advances in child development and behavior (Vol. 33, pp. 1-42). Oxford, England: Elsevier.

Siegler, R.S., \& Booth, J.L. (2004). Development of numerical estimation in young children. Child Development, 75, 428444.

Siegler, R.S., \& Opfer, J.E. (2003). The development of numerical estimation: Evidence for multiple representations of numerical quantity. Psychological Science, 14, 237-243.

Siegler, R.S., \& Ramani, G.B. (2009). Playing linear number board games - but not circular ones -improves low-income preschoolers' numerical understanding. Journal of Educational Psychology, 101, 545-560.

Siegler, R.S., \& Shipley, C. (1995). Variation, selection, and cognitive change. In T. Simon \& G. Halford (Eds.), Developing cognitive competence: New approaches to process modeling (pp. 31-76). Hillsdale, NJ: Erlbaum.

Thompson, C.A., \& Opfer, J.E. (2008). Costs and benefits of representational change: Effects of context on age and sex differences in magnitude estimation. Journal of Experimental Child Psychology, 101, 20-51.

Thompson, C.A., \& Opfer, J.E. (in press). How 15 hundred is like 15 cherries: Effect of progressive alignment on representational changes in numerical cognition. Child Development. 